\title{
On the formalism of dark energy accretion onto black- and worm-holes
}

\author{
Prado Martín-Moruno \\ Colina de los Chopos, Instituto de Matemáticas y Física Fundamental, Consejo Superior de Investigaciones Científicas, Serrano 121, 28006 Madrid, Spain
}

Received 27 September 2007; received in revised form 22 October 2007; accepted 26 October 2007

Available online 3 December 2007

Editor: A. Ringwald

\begin{abstract}
In this work a general formalism for the accretion of dark energy onto astronomical objects, black holes and wormholes, is considered. It is shown that in models with four dimensions or more, any singularity with a divergence in the Hubble parameter may be avoided by a big trip, if it is assumed that there is no coupling between the bulk and this accreting object. If this is not the case in more than four dimensions, the evolution of the cosmological object depends on the particular model.
\end{abstract}

(c) 2007 Elsevier B.V. All rights reserved.

PACS: 04.70.-s; 98.80.Cq; 95.36.+x

In recent years several cosmological models have been taken into account in order to explain the current accelerated expansion of our Universe [1]. Some of these models entail the consideration of new phenomena which may decisively influence the evolution of the universe. The most popular of them is the so-called big rip singularity [2], which is a possible doomsday for the Universe where both its size and its energy density become infinitely large. This big rip seemed to be related to the assumption that the unknown energy density which carries the accelerated expansion is phantom energy [3], i.e., it appeared in models filled with a fluid with equation of state $p=w \rho$ and $w<-1$. Fortunately, an escaping route from this doomsday was found, because in these models there are wormholes which reach an infinite size before the singularity, [4].

The consideration of a particular phantom generalised Chaplygin gas model (PGCG) in order to describe the accelerating expansion of the Universe showed that a fluid with phantom nature does not necessarily imply a big rip singularity [5] or a big trip [6]. However it has been argued that the Universe is not totally save from a catastrophic doomsday. In fact, another type of future singularity could take place. It was dubbed the big freeze singularity and entails that the energy density of the universe blows up at a finite scale factor. This singularity

E-mail address: pra@imaff.cfmac.csic.es. is present in some PGCG and some dual phantom generalised Chaplygin gas (DPGCG) models (the laters can be defined in a Randall-Sundrum type 1 scenario) [7,8]. Since in the models which show a big rip singularity, this doomsday can also be avoided by a big trip, the question is whether this new singularity, too, associates an extreme growth of wormholes. It is the aim of the present report to develop a generalized formalism to describe the dark energy accretion process for black holes and wormholes, independent of the used cosmic model and of dimensionality of the spacetime.

\section{Accretion process in four-dimensional models}

It is well known that black holes accrete dark energy in a different way as ordinary matter does. To study this process, one must consider that the dark energy density covers the whole space in a homogeneous and isotropic form. The black hole mass growth rate for an asymptotic observer can be expressed as $[9,10]$

$\dot{M}=4 \pi D M^{2}(p+\rho)$,

where $D$ is a constant of order unity, the overhead dot denotes the derivative with respect the cosmic time and $G=c=1$.

On the other hand, it has been shown that phantom models, which could also describe the current accelerating expansions of the universe, contain wormholes [11]. Phantom models are 
characterized by an equation of state parameter $w<-1$. Therefore, the accretion process onto wormholes has been also studied and a similar expression for its rate of variation of mass, has been obtained [4],

$\dot{m}=-4 \pi Q m^{2}(p+\rho)$,

with $Q$ a positive constant.

The expansion of a four-dimensional homogeneous and isotropic universe can be described by the Friedmann equations

$H^{2}=\frac{8 \pi}{3} \rho$

and

$\frac{\ddot{a}}{a}=-\frac{4 \pi}{3}(\rho+3 p)$.

On the other hand, the conservation law for a perfect fluid is

$\dot{\rho}+3 H(p+\rho)=0$,

which, taking into account Eq. (3), yields

$p+\rho=-\frac{1}{\sqrt{24 \pi}} \rho^{-1 / 2} \dot{\rho}$.

Then, for black holes, Eq. (1) can be expressed as

$\dot{M}=-\sqrt{\frac{2 \pi}{3}} D M^{2} \rho^{-1 / 2} \dot{\rho}$.

One can easily integrate this expression and obtain

$M=\frac{M_{0}}{1+\sqrt{\frac{8 \pi}{3}} D M_{0}\left(\rho^{1 / 2}-\rho_{0}^{1 / 2}\right)}$,

where the subscript " 0 " denotes current value. Following a similar pattern for wormholes, one gets

$m=\frac{m_{0}}{1-\sqrt{\frac{8 \pi}{3}} Q m_{0}\left(\rho^{1 / 2}-\rho_{0}^{1 / 2}\right)}$.

This expression was already obtaining for the case in which the perfect fluid is a particular phantom generalised Chaplygin gas (PGCG) [6], though in the present case Eqs. (9), (8) also does, is valid for any homogeneous and isotropic model in four dimensions. Taking into account Eq. (3), both mass functions can be expressed in terms of the Hubble parameter [12]

$M(t)=\frac{M_{0}}{1+D M_{0}\left[H(t)-H_{0}\right]}$

and

$m(t)=\frac{m_{0}}{1-Q m_{0}\left[H(t)-H_{0}\right]}$.

Thus, the black hole mass will increase if and only if the Hubble parameter decreases. Then, since $\dot{H}=\ddot{a} / a-H^{2}=$ $-4 \pi(p+\rho), p+\rho>0$, a conclusion which can also be directly extracted from Eq. (1). It follows that in order to infinitely increase the mass in a finite time, the black hole must have a current mass $M_{0}>1 /\left(D H_{0}\right) \sim 10^{23} M_{\odot}$, and that is not possible [10]. This mass will decrease in phantom models, $p+\rho<0$, vanishing where $H(t)$ diverges, in case that this eventually happens. On the other hand, a similar argument shows that the wormhole mass will increases only in phantom models, where the wormhole throat became infinitely large at $t_{*}$, with $H\left(t_{*}\right)=H_{0}+1 /\left(Q m_{0}\right)<\infty$, if the universe reaches that time. But, if there is a singularity in the model in which the Hubble parameter is infinity, since the Hubble parameter is an ever increasing function, then $t_{0}<t_{*}<t_{\text {sing }}$. Therefore the wormhole would have an infinitely large size before the universe reaches the singularity, that singularity occurring at a finite or infinite scale factor, i.e., a big trip could avoid all the singularities with an infinite Hubble parameter that could appear in any homogeneous and isotropic four-dimensional model.

Now we consider the particular case of phantom generalised Chaplygin gas models. As it is well known, generalised Chaplygin gas (GCG) satisfies the equation of state [13]

$p=-\frac{A}{\rho^{\alpha}}$,

where $A$ is a constant and $\alpha$ is a parameter (corresponding to the case $\alpha=1$ in the original Chaplygin gas). The conservation law for the energy momentum tensor for this fluid can be expressed as

$\rho=\left(A+\frac{B}{a^{3(1+\alpha)}}\right)^{\frac{1}{1+\alpha}}$,

with $B$ a constant (which can be expressed in terms of $A, \rho_{0}$ and $\left.a_{0}\right)$. Such a fluid must fulfill $\rho>0$ and $p+\rho<0$ in order to have a phantom nature, $[5,14]$. It can be shown [8], that there are four different kinds of phantom generalised Chaplygin gas (PGCG) models, corresponding to four different universes. The cosmological scenarios fulfilling $1+\alpha>0$ are asymptotically de Sitter in the future. The two models with $1+\alpha<0$, show a big freeze singularity at a finite time in the future, i.e., the scale factor grows to a maximum finite value where the energy density diverges. These singular models correspond to $A>0$, $B<0$ and $1+\alpha<0$, and $A<0, B>0$ and $(1+\alpha)^{-1}=2 n<0$ (with $n$ some negative integer number), respectively, and the maximum scale factor in both cases is

$a_{\max }=\left|\frac{B}{A}\right|^{\frac{1}{3(1+\alpha)}}$.

As this singularity implies a divergence on the Hubble parameter a big trip phenomenon should take place, as we have argued before. In fact, if one defines $x=a / a_{\max }(0 \leqslant x \leqslant 1)$, Eq. (11) for the first mentioned case can be re-expressed as

$m(x)=\frac{m_{0}}{1+\sqrt{\frac{8 \pi}{3}} \frac{Q m_{0}}{|A|^{n}}\left[\frac{1}{\left(1-x_{0}^{3 p}\right)^{n}}-\frac{1}{\left(1-x^{3 p}\right)^{n}}\right]}$.

It is the same for the second considered case replacing $|A|$ for $A$ and $n$ for $-1 /[2(1+\alpha)]$. Since $m(0)<m_{0}$ and $m_{0}>m(1)$, either the function $m(x)$ changes from an increasing function to a decreasing function in the $\mathrm{x}$-interval $(0,1)$, or it diverges at least once on this interval. If one defines $F(x)=m_{0} / m(x)$, it is easy to see that $F(x)$ is a continuous and increasing function in the whole interior of this interval. Therefore $m(x)$ is an increasing function with $m_{0}>m(1)$, so it must diverge in $\left(x_{0}, 1\right)$. 
Then the wormhole mass and throat size will be infinitely large before the universe reaches its doomsday (at $x=1$ ), i.e., these universes present a big trip which could avoid the big freeze singularity as it was expected.

\section{Accretion in higher dimensional models}

If we consider cosmological models constructed in a space with more than four dimensions, the Friedmann equation would read

$H^{2}=\frac{8 \pi}{3} F(\rho)$

where $F(\rho)$ is a generic function of $\rho, \rho$ being the dark energy density which is defined on the bulk space. It is well known that the correction introduced in Eq. (3) which leads to Eq. (16) must be relevant only at hight energies [15]. Since in models with $w+1<0$ the energy density increases with time, this correction could even be dominant at the late time acceleration period. Moreover, in this work we consider dual models which imply an absolute value of the energy density sufficiently large to do this correction indispensable.

As we only see four dimensions, we could treat that as if the expansion of our universe was originated by a effective $\rho_{\mathrm{eff}}=F(\rho)$, which is the projection of $\rho$ onto four dimensions. Therefore, in order to consider the accretion process there are two possibilities. Firstly, when the astronomical object can accrete the whole fluid on the bulk or, secondly, when the object placed in the four-dimensional patch only accretes the effective fluid which can be defined on these dimensions.

\subsection{Accretion of the fluid on the bulk}

Here we will assume that there is some coupling between the bulk and the brane, allowing the astronomical object to accrete the fluid which drives the physics of the universe, as it is done in [12] for a particular model. Considering Eq. (16) and the conservation law Eq. (5), we can obtain

$p+\rho=-\frac{1}{\sqrt{24 \pi}} \rho_{\text {eff }}^{-1 / 2} \dot{\rho}$,

and inserting this expression in Eq. (1)

$\frac{\mathrm{d} M}{M^{2}}=-\sqrt{\frac{2 \pi}{3}} D \rho_{\mathrm{eff}}^{-1 / 2} \mathrm{~d} \rho$.

In order to integrate this expression one must know the specific functional form of $F(\rho)$. Therefore the black hole mass function will depend on the considered cosmological model and the same conclusion can be extracted in the wormhole case.

A Randall-Sundrum type 1 scenario, [16], is a five-dimensional model with a four-dimensional brane which could represent our Universe. The scale factor in the four-dimensional universe can be defined with the modified from Friedmann equation on the brane

$H^{2}=\frac{8 \pi}{3} \rho\left(1+\frac{\rho}{2 \lambda}\right)$, where $\lambda$ is the positive brane tension and $\rho$ the energy density on the bulk. In this scenario, one can consider a phantom fluid, $w<-1$, which fulfill the null energy condition, $p+\rho>0$, and has a well defined Hubble parameter; this fluid is known as dual phantom fluid [12]. That would implied an energy density negative definite, with $\rho<-2 \lambda$, but it must be also noticed that the effective energy density on the brane keeps always being positive.

A dual phantom generalised Chaplygin gas (DPGCG) is defined as a perfect fluid satisfying Eq. (13), with the abovementioned characteristics $[7,8]$. These characteristics are satisfied provided that $A<0$ and $B>0$ in Eq. (13). If one also considers $(1+\alpha)^{-1}=2 n+1$ with $n$ a negative integer, two models are obtained which show a big freeze singularity in the future. In these models Eqs. (12), (13) and (19) imply

$p+\rho=\frac{x^{\frac{3}{1+2 p}}}{|A|^{1+2 p}\left(1-x^{\frac{3}{1+2 p}}\right)^{2(1+p)}}$,

where $1+2 p=-(1+2 n)$ with $x<1$ and no-negative $(x=1$ corresponding to the big freeze). Replacing Eq. (13) in Eq. (19), one obtains

$\dot{x}=\sqrt{\frac{8 \pi}{3}} x \frac{\left[1-2 \lambda|A|^{1+2 p}\left(1-x^{\frac{3}{1+2 p}}\right)^{1+2 p}\right]^{1 / 2}}{\sqrt{2 \lambda}|A|^{1+2 p}\left(1-x^{\frac{3}{1+2 p}}\right)^{1+2 p}}$.

Taking into account Eqs. (20) and (21), one can integrate Eq. (2) to yield

$m(k)=\frac{m_{0}}{1+\sqrt{\frac{4 \pi \lambda}{3}} Q m_{0}\left[\operatorname{Ln}\left(\frac{1+k}{1-k}\right)-\operatorname{Ln}\left(\frac{1+k_{0}}{1-k_{0}}\right)\right]}$,

with $k=\left[1-2 \lambda|A|^{1+2 p}\left(1-x^{\frac{3}{1+2 p}}\right)^{1+2 p}\right]^{1 / 2}$.

Therefore, when $a$ increases, $k$ increases and vice versa. The $\operatorname{Ln}[(1+k) /(1-k)]$ is a positive increasing function in the whole interval, because $(1+k) /(1-k)>1$ for these values. This implies that the wormhole mass will decrease when the scale factor increases; in fact, replacing the value $x=1$ in the above expression, one can see that the wormhole tends to disappear at the singularity.

On the other hand, it is shown that in some dual phantom models [12] a big hole phenomenon could take place, i.e., a black hole could grow up so rapidly that its size could become infinitely large before the singularity. To see if that is the case we must integrate the black hole mass rate Eq. (1) for an asymptotic observer. In these models this integration produces

$$
M=\frac{M_{0}}{1+\sqrt{\frac{4 \pi \lambda}{3}} D M_{0}\left[\operatorname{Ln}\left(\frac{1+k_{0}}{1-k_{0}}\right)-\operatorname{Ln}\left(\frac{1+k}{1-k}\right)\right]} .
$$

The $\operatorname{Ln}$ takes values $0 \leqslant \operatorname{Ln}[(1+k) /(1-k)] \leqslant \infty$ for $k$ in $[0,1]$, so there must be some $k_{0}<k_{*}<1$ for which the denominator of Eq. (23) vanishes; so this expression diverges before the universe reaches its maximum size. Therefore a black hole would increase its size so rapidly that would eventually engulf the universe itself, which always has a finite size, before it reaches the singularity. 


\subsection{Accretion of the fluid on the brane}

If the considered object accretes the fluid that is defined in the same dimensions as it, as studied in [17] for a particular case, then in Eqs. (1) and (2) we must have $\rho_{\text {eff }}+p_{\text {eff. Differ- }}$ entiating Eq. (16) one obtains

$2 H \dot{H}=\frac{8 \pi}{3} \frac{\mathrm{d} \rho_{\mathrm{eff}}}{\mathrm{d} \rho} \dot{\rho}$,

then, using the conservation law given by Eq. (5),

$\dot{H}=-4 \pi \frac{\mathrm{d} \rho_{\mathrm{eff}}}{\mathrm{d} \rho}(p+\rho)$.

In order to define an effective pressure, we take

$\frac{\ddot{a}}{a}=\dot{H}+H^{2}=-\frac{4 \pi}{3}\left(\rho_{\text {eff }}+3 p_{\text {eff }}\right)$,

which implies

$p_{\text {eff }}+\rho_{\text {eff }}=(p+\rho) \frac{\mathrm{d} \rho_{\text {eff }}}{\mathrm{d} \rho}$.

Now Eq. (1) can be expressed as

$\frac{\mathrm{d} M}{M^{2}}=4 \pi D(p+\rho) \frac{\mathrm{d} \rho_{\text {eff }}}{\mathrm{d} \rho} \mathrm{d} t$,

which, taking into account Eqs. (5) and (16), yields

$\frac{\mathrm{d} M}{M^{2}}=-\sqrt{\frac{2 \pi}{3}} D \rho_{\mathrm{eff}}^{-1 / 2} \mathrm{~d} \rho_{\mathrm{eff}}$.

This expression can be easily integrated to obtain the black hole mass

$M=\frac{M_{0}}{1+\sqrt{\frac{8 \pi}{3}} D M_{0}\left(\rho_{\text {eff }}^{1 / 2}-\rho_{\text {effo }}^{1 / 2}\right)}$

and following a similar way

$m=\frac{m_{0}}{1-\sqrt{\frac{8 \pi}{3}} Q m_{0}\left(\rho_{\mathrm{eff}}^{1 / 2}-\rho_{\mathrm{eff} 0}^{1 / 2}\right)}$,

for the wormhole mass. We must also point out that these expressions are the same as Eq. (8), replacing $\rho$ by $\rho_{\text {eff }}$, the energy density defined in our universe. In fact, one can easily see that in terms of the Hubble parameter one obtains the same expressions as in the four-dimensional case

$M=\frac{M_{0}}{1+D M_{0}\left[H(t)-H_{0}\right]}$

and

$m=\frac{m_{0}}{1-Q m_{0}\left[H(t)-H_{0}\right]}$.

As in Section 1, the black hole mass is an increasing function if the Hubble parameter is a decreasing function, but now that occurs if $p+\rho>0$ and $\mathrm{d} \rho_{\mathrm{eff}} / \mathrm{d} \rho>0$ or if $p+\rho<0$ and $\mathrm{d} \rho_{\text {eff }} / \mathrm{d} \rho<0$, both cases corresponding to an effective dark energy fluid with $w_{\text {eff }}>-1$. A big hole phenomenon is not possible because, as it was mentioned before, there cannot be black holes with the necessary initial mass in our Universe. On the other hand, wormholes will increase if the effective fluid is a phantom fluid, which occurs if $p+\rho>0$ and $\mathrm{d} \rho_{\text {eff }} / \mathrm{d} \rho<0$ or if $p+\rho<0$ and $\mathrm{d} \rho_{\text {eff }} / \mathrm{d} \rho>0$. In both cases the fluid will be phantom or not on the bulk depending on the specific scenario. If in the model there is a singularity where the Hubble parameter tends to infinity, the black holes would disappear at this singularity. And because $H_{0}$ is finite and the Hubble parameter is a continuous function, there must necessarily be a time $t_{0}<t_{*}<t_{\text {sing }}$ defined for $H\left(t_{*}\right)=H_{0}+1 /\left(Q m_{0}\right)$, where the wormhole will become infinitely large; therefore the universe does not reach the singularity because a big trip phenomenon would take place before.

In a Randall-Sundrum type I scenario, the effective energy density and pressure on the brane can be defined

$\rho_{\text {eff }}=\rho\left(1+\frac{\rho}{2 \lambda}\right)$

and

$p_{\text {eff }}=p\left(1+\frac{\rho}{\lambda}\right)+\frac{\rho^{2}}{2 \lambda}$,

so that $w_{\text {eff }}=p_{\text {eff }} / \rho_{\text {eff }}$, and one has

$1+w_{\text {eff }}=(1+w) \frac{1+\rho / \lambda}{1+\rho /(2 \lambda)}$.

Therefore, for this scenario with an arbitrary homogeneous and isotropic fluid on the bulk (not necessarily a generalised Chaplygin gas), the sign of $1+w_{\text {eff }}$ is the same as the sign of $1+w$. Then in the DPGCG models described in the previous subsection, we have an effective phantom fluid in the brane with, obviously, $\rho_{\text {eff }}>0$. Moreover, as the fraction in Eq. (36) is always positive and bigger than $2,1+w_{\text {eff }}$ is more negative than $1+w$, i.e., it is "more phantom".

In the models mentioned above, Eq. (33) yields

$m(k)=\frac{m_{0}}{1+\sqrt{\frac{16 \pi \lambda}{3}} Q m_{0}\left(\frac{k_{0}}{1-k_{0}^{2}}-\frac{k}{1-k^{2}}\right)}$,

where $k$ is the same as in the previous subsection. One can define $f(k)=k /\left(1-k^{2}\right)$. As $f\left(k_{0}\right)<f(k)<\infty$ for $x_{0}<x<1$, there is a $k_{*}<\infty$ (which implies $x_{*}<1$ ) with $f\left(k_{*}\right)=f\left(k_{0}\right)+$ $\left(\sqrt{\frac{16 \pi \lambda}{3}} Q m_{0}\right)^{-1}$, where the wormhole mass diverges. Therefore, a big trip would occur as one would expect under general grounds.

\section{Conclusions}

In this work we have seen that in four-dimensional, homogeneous and isotropic models, black holes grow up if the Hubble parameter is a decreasing function, which implies $w>-1$; otherwise, they would shrink down to zero in a finite time in the presence of a singularity with divergent $H(t)$, or asymptotally if there is no such a future singularity. It must be also pointed out that in dark GCG (DGCG) models with $0<\alpha<1$, generalised Chaplygin dark stars should exist that would increase their size with time [18]. Since this is a dark model, $w>-1$, black holes should also grow, as it could be expected from the behaviour of the dark stars. On the other hand, wormholes would shrink 
if $H(t)$ decreases and increase its size if $H(t)$ is an increasing function. In fact, in models which show a future singularity with divergence in the Hubble parameter (which in these models means a divergence in the energy density), this singularity would be avoided by a big trip, because there is a time $t_{*}$ before the singularity where the wormhole would be infinitely large. In particular, we have shown that this big trip occurs in PGCG models also with a big freeze.

The case of models with more than four dimensions is a little more complicated, because it depends on whether the object could accrete the whole fluid on the bulk or accretes only the effective fluid which can be defined on the brane where the wormhole is. In the first case, we have shown that the mass function will depend on the model considered. In the DPGCG case, wormholes decrease their size when the scale factor increases, so a big trip phenomenon is not possible. But, in this case, black holes grow up and can engulf the universe before the singularity. In the second case, we consider that the object is able to accrete only the effective fluid, so that the conclusions are the same as in four-dimensional models, i.e., any singularity implying a divergence of the Hubble parameter that could take place at a finite time in the future is avoided by a big trip. In DPGCG models the results obtained following the above two ways are the opposite, since though in the first case the universe avoids a doomsday, it may find an even more dramatic end. In the second case the universe embarks itself in a trip with unknown destination. Besides physical considerations, simplicity and beauty dictate that, for cosmological models with a modified Friedmann equation, the accretion process is most appropriately dealt with by the considering accretion of the effective fluid with four-dimensional energy density. If we take into account recent work by Frampton [19] who argued that effective energy density and pressure are not proper physical quantities, it appears that one ought to choose the former of the above two interpretations.

\section{Acknowledgements}

The author thanks Pedro F. González Díaz for useful discussions and encouragement and Marco Tripodi for valuable technical help and creating a wonderful working atmosphere. This work was supported by MEC under Research Project No. FIS2005-01181. The author gratefully acknowledges the financial support provided by the I3P framework of CSIC and the European Social Fund.

\section{References}

[1] E.J. Copeland, M. Sami, S. Tsujikawa, Int. J. Mod. Phys. D 15 (2006) 1753, hep-th/0603057.

[2] R.R. Caldwell, M. Kamionkowski, N.N. Weinberg, Phys. Rev. Lett. 91 (2003) 071301, astro-ph/0302506;

P.F. González-Díaz, Phys. Lett. B 586 (2004) 1, astro-ph/0312579; P.F. González-Díaz, Phys. Rev. D 69 (2004) 063522, hep-th/0401082; S. Nojiri, S.D. Odintsov, Phys. Rev. D 70 (2004) 103522, hep-th/0408170.

[3] R.R. Caldwell, Phys. Lett. B 545 (2002) 23, astro-ph/9908168;

V.K. Onemli, R.P. Woodard, Class. Quantum Grav. 19 (2002) 4607, grqc/0204065;

S.M. Carroll, M. Hoffman, M. Trodden, Phys. Rev. D 68 (2003) 023509, astro-ph/0301273.

[4] P.F. González-Díaz, Phys. Rev. Lett. 93 (2004) 071301, astro-ph/0404045; P.F. González-Díaz, Phys. Lett. B 635 (2006) 1, hep-th/0607137; P.F. González-Díaz, P. Martín-Moruno, arXiv: 0704.1731 [astro-ph], Proceedings of the MG11, in press.

[5] M. Bouhmadi-López, J.A. Jiménez Madrid, JCAP 0505 (2005) 005, astro$\mathrm{ph} / 0404540$.

[6] J.A. Jiménez Madrid, Phys. Lett. B 634 (2006) 106, astro-ph/0512117.

[7] M. Bouhmadi-López, P.F. González-Díaz, P. Martín-Moruno, gr-qc/ 0612135 .

[8] M. Bouhmadi-López, P.F. González-Díaz, P. Martín-Moruno, arXiv: 0707.2390 [gr-qc].

[9] E. Babichev, V. Dokuchaev, Yu. Eroshenko, Phys. Rev. Lett. 93 (2004) 021102, gr-qc/0402089;

E. Babichev, V. Dokuchaev, Y. Eroshenko, J. Exp. Theor. Phys. 100 (2005) 528, Zh. Eksp. Teor. Fiz. 127 (2005) 597, astro-ph/0505618;

E. Babichev, V.F. Mukhanov, A. Vikman, JHEP 0609 (2006) 061, hep-th/ 0604075;

J.A. de Freitas Pacheco, J.E. Horvath, arXiv: 0709.1240 [gr-qc].

[10] P. Martín-Moruno, J.A. Jiménez Madrid, P.F. González-Díaz, Phys. Lett. B 640 (2006) 117, astro-ph/0603761.

[11] S.V. Sushkov, Phys. Rev. D 71 (2005) 043520, gr-qc/0502084; F.S.N. Lobo, Phys. Rev. D 71 (2005) 084011, gr-qc/0502099.

[12] A.V. Yurov, P. Martín-Moruno, P.F. González-Díaz, Nucl. Phys. B 759 (2006) 320, astro-ph/0606529.

[13] A.Y. Kamenshchik, U. Moschella, V. Pasquier, Phys. Lett. B 511 (2001) 265, gr-qc/0103004;

N. Bilić, G.B. Tupper, R.D. Viollier, Phys. Lett. B 535 (2002) 17, astro$\mathrm{ph} / 0111325$;

M.C. Bento, O. Bertolami, A.A. Sen, Phys. Rev. D 66 (2002) 043507, gr-qc/0202064.

[14] I.M. Khalatnikov, Phys. Lett. B 563 (2003) 123.

[15] J.M. Cline, C. Grojean, G. Servant, Phys. Rev. Lett. 83 (1999) 4245, hep$\mathrm{ph} / 9906523$;

M.C. Bento, O. Bertolami, A.A. Sen, Phys. Rev. D 67 (2003) 023504, gr-qc/0204046.

[16] L. Randall, R. Sundrum, Phys. Rev. Lett. 83 (1999) 4690, hep-th/9906064.

[17] X. Zhang, arXiv: 0708.1408 [gr-qc].

[18] O. Bertolami, J. Paramos, Phys. Rev. D 72 (2005) 123512, astro-ph/ 0509547.

[19] P.H. Frampton, arXiv: 0709.1630 [gr-qc]. 